\title{
Association of vascular calcification of breast with carotid intima media thickness
}

\author{
Priya P. Sankaran ${ }^{A, B, C, D, E, F, G}$, Prajeeth Rao N. ${ }^{A, B, C, D, E, F}$, Prakashini Koteshwara ${ }^{F}$ \\ Department of Radiodiagnosis and Imaging, Kasturba Medical College, Manipal, Karnataka, India
}

\section{Abstract}

\begin{abstract}
Purpose: To assess the relationship between breast arterial calcification (BAC) on mammography and carotid intima medial thickness (C-IMT) by comparing these patients with healthy control subjects.

Material and methods: The study included 100 women divided into two groups based on presence of BAC on mammography using the WIPRO GE - DMR PLUS mammography unit. The groups included $50 \mathrm{BAC}(+)$ (case group) and $50 \mathrm{BAC}(-)$ (control group). BAC is defined as two linear calcific deposits forming the rail-road track pattern, with ringlike appearance in cross section. Relevant previous clinical and surgical history was taken and C-IMT was measured using B-mode ultrasonography Aplio XG (Toshiba medical systems corp., Japan) using an 8-13 MHz linear transducer within $1 \mathrm{~cm}$ from the proximal and distal portion of the common carotid artery and the mean value was taken.

Results: All the participants included in this study were within the age group of 40-70 years. Mean age with $\mathrm{BAC}(+)$ was $59.18 \pm 8.59$ years and BAC $(-)$ was $50.70 \pm 7.93$ years. Mean C-IMT in the BAC $(+)$ group was $0.86 \pm 0.21 \mathrm{~mm}$ vs. $0.71 \pm 0.12 \mathrm{~mm}$ in the BAC(-) group. Chi-square test, independent $t$ test and Spearman's rho correlation to assess the association of the breast arterial calcification were performed. The study showed significant difference in mean C-IMT between two groups ( $p$ value $<0.001$ ) when correlated with age, diabetes, hypertension, presence of BAC and history of surgery and drug intake.
\end{abstract}

Conclusions: BAC in mammography is independently associated with C-IMT. Furthermore, apart from age and menopausal status a statistically significant positive correlation with C-IMT was observed.

Key words: breast arterial calcification, carotid intima media thickness, mammography.

\section{Introduction}

Screening mammography is recommended in women over the age of 40 years for early detection of breast cancer in which breast arterial calcification (BAC) is an incidental finding [1].

Mönkeberg medial calcific sclerosis is the underlying pathology leading to BAC, which is detected at mammography as linear, parallel lines [2] resembling arail road appearance or with ring-like appearance on cross section [3].

Atherosclerosis is the result of hyperlipidemia and lipid oxidation which have been the major cause of cardiovascular mortality in developed countries. It affects the intima of blood vessels and is characterized by formation of plaques [4]. It starts in childhood and progresses gradually with age at varying rates, leading to susceptibility to complications.

Atherosclerosis can be identified on imaging as arterial calcifications in radiographs and computed tomography (CT) and in the form of an increase in intima medial thickness (IMT) on ultrasound and contrast enhanced CT.

Carotid intima-medial thickness (C-IMT) is quickly and easily measured and is a reproducible [5] ultrasonographic marker of early atherosclerotic disease [6-9] beyond classic cardiovascular and cerebrovascular risk factors, and is useful in predicting clinical events in the

\section{Correspondence address:}

Dr. Prajeeth Rao N, Department of Radiodiagnosis and Imaging, Kasturba Medical College, Manipal, Karnataka 5761014, India, e-mail: prajithng@gmail.com Authors' contribution:

A Study design · B Data collection · C Statistical analysis · D Data interpretation · E Manuscript preparation · F Literature search · G Funds collection 
Table 1. Risk factors comparison between $B A C(+)$ and $B A C(-)$ groups

\begin{tabular}{|c|c|c|c|c|c|c|}
\hline & \multicolumn{4}{|c|}{ Vascular calcifications } & \multirow{3}{*}{$\begin{array}{l}\chi^{2}, \mathrm{df}, \\
p \text {-value }\end{array}$} \\
\hline & & \multicolumn{2}{|c|}{$B A C+v e$} & \multicolumn{2}{|c|}{$B A C-v e$} & \\
\hline & & Count & $\%$ & Count & $\%$ & \\
\hline \multirow[t]{2}{*}{ Diabetes } & No & 33 & 66.0 & 39 & 78.0 & \multirow{2}{*}{$\begin{array}{c}1.786,1, \\
0.181\end{array}$} \\
\hline & Yes & 17 & 34.0 & 11 & 22.0 & \\
\hline \multirow[t]{2}{*}{ Hypertension } & No & 27 & 54.0 & 36 & 72.0 & \multirow{2}{*}{$\begin{array}{c}3.475,1, \\
0.062\end{array}$} \\
\hline & Yes & 23 & 46.0 & 14 & 28.0 & \\
\hline \multirow{2}{*}{$\begin{array}{l}\text { Menopause } \\
\text { attained }\end{array}$} & No & 6 & 12.0 & 25 & 50.0 & \multirow{2}{*}{$\begin{array}{l}16.877,1 \\
<0.001\end{array}$} \\
\hline & Yes & 44 & 88.0 & 25 & 50.0 & \\
\hline
\end{tabular}

Table 2. Vascular calcifications (VAC) grading in $\mathrm{BAC}(+)$ group

\begin{tabular}{|l|c|c|}
\hline \multirow{2}{*}{ VAC grading } & \multicolumn{2}{|c|}{ BAC(+) } \\
\cline { 2 - 3 } & Count & $\%$ \\
\hline Grade 0 & 0 & 0.0 \\
\hline Grade 1 & 20 & 40.0 \\
\hline Grade 2 & 17 & 34.0 \\
\hline Grade 3 & 13 & 26.0 \\
\hline
\end{tabular}

Table 3. Showing comparison of different variables in $\mathrm{BAC}(+)$ and $\mathrm{BAC}(-)$ subjects

\begin{tabular}{|l|c|c|c|}
\hline Parameters & $\mathrm{BAC}(+)$ & $\mathrm{BAC}(-)$ & $p$-value \\
\hline Age (years) & $59.18 \pm 8.59$ & $50.70 \pm 7.93$ & $<0.001$ \\
\hline Diabetes mellitus & $17(34 \%)$ & $11(22 \%)$ & 0.181 \\
\hline Hypertension & $23(46 \%)$ & $14(28 \%)$ & 0.062 \\
\hline Menopausal status & $44(88 \%)$ & $25(50 \%)$ & $<0.001$ \\
\hline Surgical history & $1(2 \%)$ & 0 & 0.315 \\
\hline C-IMT & $0.86 \pm 0.21$ & $0.71 \pm 0.12$ & $<0.001$ \\
\hline
\end{tabular}

near future, including myocardial infarction, stroke and mortality due to cerebrovascular disease.

Previous studies revealed the relationship between breast arterial calcification and coronary artery disease [10] hypertension [11], diabetes mellitus [12], carotid plaques [13], dyslipidemia [14], visceral obesity [14] and chronic kidney disease [15]. Studies assessing the relationship between breast arterial calcification and carotid intima medial thickness have been limited [16,17]. Therefore, our study was designed to assess the relationship between breast arterial calcification on mammography and carotid intima medial thickness.

\section{Material and methods}

This is a hospital-based time-bound prospective study, conducted for a period of 12 months between September 2017 and August 2018 in the Department of Radiodiagnosis and Imaging, Kasturba Medical College, Manipal.

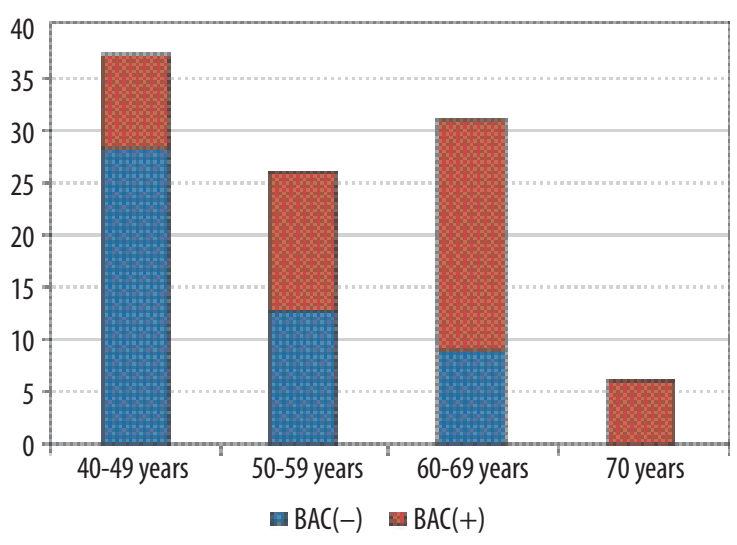

Figure 1. Bar diagram showing age distribution of patients with both $B A C(+)$ and $B A C(-)$

Institutional ethics committee approval (IEC:529/2017) was obtained. Informed consent was taken from every patient.

A total of 100 women who underwent screening mammograms with the WIPRO GE - DMR PLUS mammography unit were included in the study. These subjects were divided into two groups based on the presence of breast arterial calcifications in screening mammograms. The subjects who were found to have breast arterial calcifications were labeled as cases and subjects without breast arterial calcifications were labeled as controls.

Relevant clinical history was recorded regarding the past history of diabetes mellitus, hypertension, drug intake (ingestion of lipid-lowering drugs), menopausal status and previous surgical history.

Standard medio-lateral oblique (MLO) and craniocaudal (CC) projections were obtained. Additional projections, such as coned-down compression and magnification views, were considered when further help in analysis was expected.

Breast arterial calcification on mammography is defined as two linear calcific deposits forming the rail-road track pattern, with ring-like appearance in cross section. Furthermore, these vascular calcifications are graded based on their appearance into 3 main grades:

- grade 1 , faintly outlined by calcium,

- grade 2, predominantly outlined by calcium,

- grade 3, markedly outlined by calcium [18].

For statistical purposes subjects without breast arterial calcification were graded as grade 0 .

Carotid intima medial thickness was assessed on an Aplio XG (Toshiba medical systems corp., JAPAN) using an $8-13 \mathrm{MHz}$ linear transducer. The patient is positioned in a supine position with a pillow at the level of the shoulders of the patient in order to hyperextend the neck and the patient's neck is turned away from the side of examination by 45 degrees and the transducer is placed along the sagittal axis of the carotid vessels.

IMT of both carotids was measured using a caliper in $B$ mode ultrasound. Both intima (echogenic layer) and media (echo poor) were included in measurement [19]. 
C-IMT was measured at four different levels, at the proxi$\mathrm{mal}$ and distal portions within $1.0 \mathrm{~cm}$ length from the origin and proximal to the bifurcation in both carotid arteries and the mean of these four measurements were recorded.

\section{Statistical analysis}

Data were entered into a Microsoft Excel data sheet and were analyzed using SPSS version 22 (IBM SPSS Statistics, Somers NY, USA) software. Categorical data were presented in the form of frequencies and proportions. The $\chi^{2}$ test was used as a test of significance for qualitative data.

Continuous data were presented as mean and standard deviation. The independent $t$ test was used as a test of significance to identify the mean difference between two quantitative variables.

Graphical representation of data: MS Excel and MS Word were used to obtain various types of graphs such as bar diagram, pie diagram and scatter plots.

The $p$ value (probability that the result is true) of $<0.05$ was considered as statistically significant after assuming all the rules of statistical tests.

We used Spearman's rho correlation to assess the association of breast arterial calcification with carotid intima medial thickness and also to assess the relationship between the grade of arterial calcification with the carotid intima medial thickness.

\section{Results}

In the study a total of 100 subjects $50 \mathrm{BAC}(+)$ and $50 \mathrm{BAC}(-)$ with the age range $40-70$ years were included. The maximum number of subjects was in the age group of 40-49 years $(n=37)$ and the minimum in the age group of 70 years $(n=7)$ (Figure 1).

The mean age of $\mathrm{BAC}(+)$ was $59.18 \pm 8.59$ years with $34 \%$ being diabetic, $46 \%$ being hypertensive and $88 \%$ having attained menopause. In the BAC(-) group the mean age of BAC(-) was $50.70 \pm 7.93$ years, with $22 \%$ being diabetics, $28 \%$ being hypertensive and $50 \%$ of them having attained menopause (Table 1$)$.

Mean C-IMT in BAC(+) was $0.86 \pm 0.21 \mathrm{~mm}$ and that of BAC (-) group was $0.71 \pm 0.12 \mathrm{~mm}$, showing a statistically significant difference $(p<0.05)$ in mean C-IMT between the two groups. Apart from BAC, mean age of the population and menopause showed a significant difference $(p<0.05)$ with respect to increase in C-IMT between both groups.

Grading of the calcification was performed in the $\mathrm{BAC}+$ ve group with grade 1 in $40 \%$, grade 2 in $34 \%$ and grade 3 in $26 \%$ of the population. When BAC grading was assessed with C-IMT there was a linear positive correlation, i.e. with increase in grade of breast arterial calcification (BAC), there was an increase in C-IMT and vice versa but this increase was statistically insignificant (Table 2, Figures 2-4).

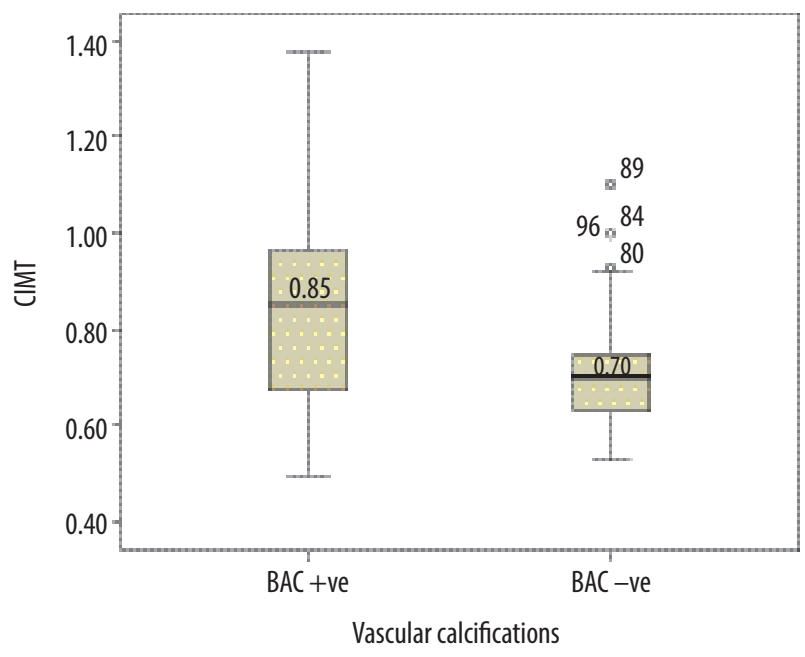

Figure 2. Box plot showing median C-IMT distribution comparison with respect to vascular calcifications

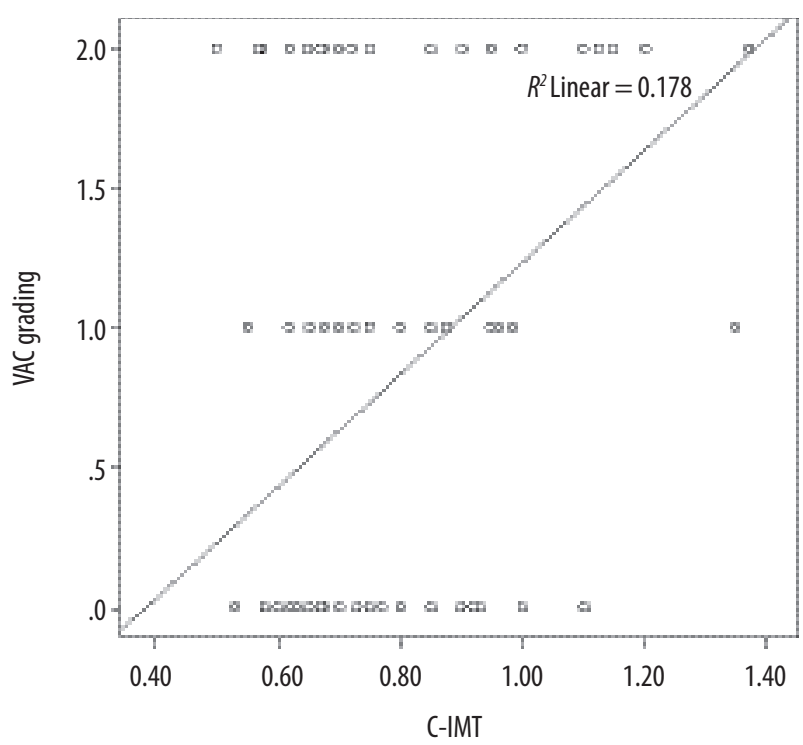

Figure 3. Scatter plot showing Spearman's rho correlation between VAC grading and C-IMT

In the study there was a significant positive correlation between age and C-IMT (overall age, age in BAC +ve group and age in BAC -ve group), i.e. with increase in age there was an increase in C-IMT and vice versa (Table 3 ).

\section{Discussion}

The present study showed that the presence of BAC was associated with increased intima media thickness of the carotid arteries. Apart from the C-IMT, age and menopausal status showed a significant positive correlation.

Atherosclerosis is the result of hyperlipidemia and lipid oxidation, which has been the major cause of cardiovascular mortality in developed countries. It affects the intima of blood vessels and all the vessels from the aorta to the coronary arteries can be involved, being characterized by intimal plaques [20]. It begins in childhood and 

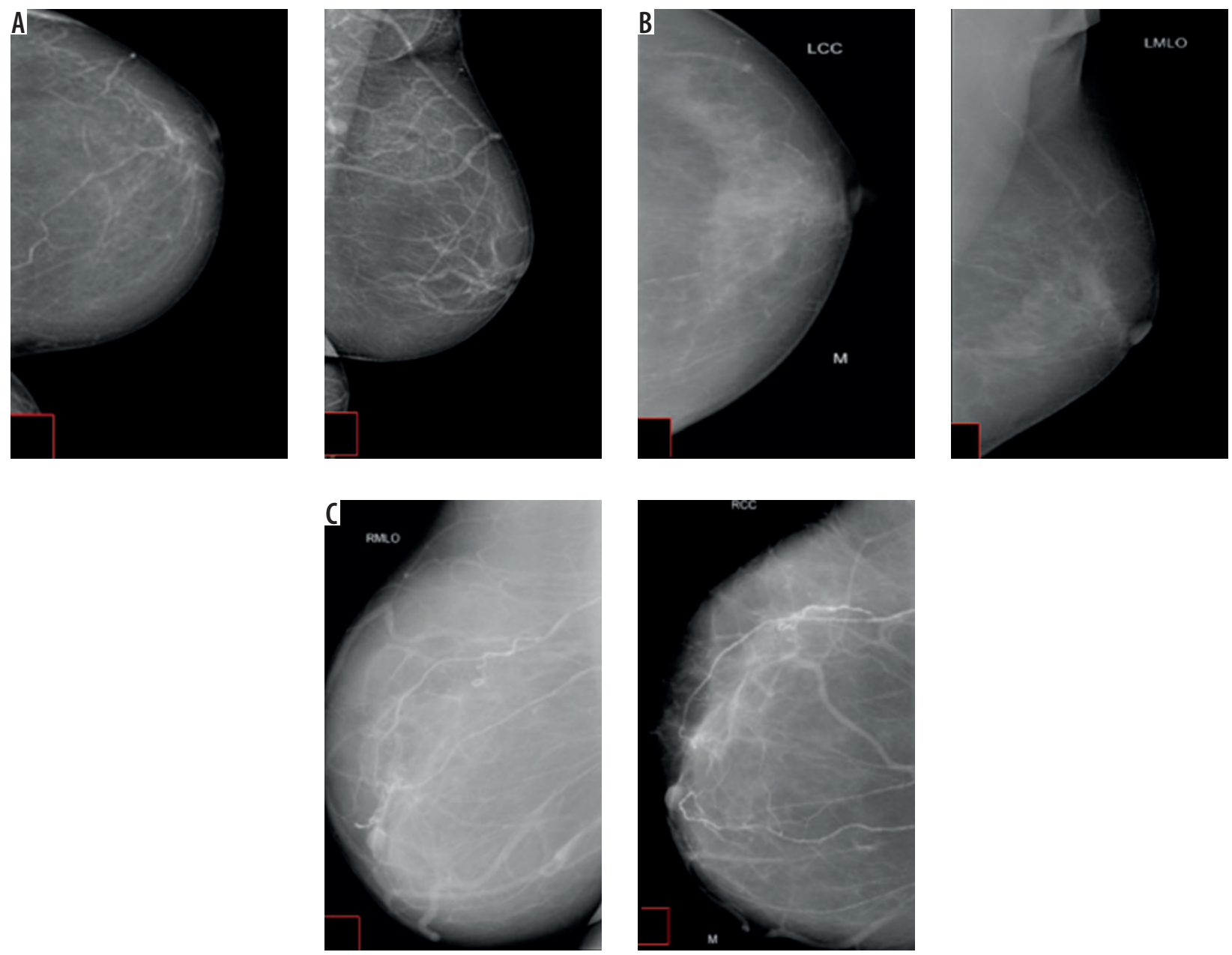

Figure 4. A) Screening mammogram in 60-year-old woman showing linear calcifications outlined by calcium, suggesting grade I breast arterial calcifications. B) Screening mammogram in 67-year-old woman showing linear calcifications which are predominantly outlined by calcium, suggesting grade II breast arterial calcifications. C) Screening mammogram of 65-year-old woman showing linear calcifications which are markedly outlined by calcium, suggesting grade III breast arterial calcifications

progresses gradually with age at varying rates, leading to susceptibility to complications.

Atherosclerosis can be identified on imaging as arterial calcifications in radiographs and CT and in the form of an increase in intima medial thickness (IMT) on ultrasound.

Arterial calcifications are divided into two groups based on the involved layer.

1. Intimal calcifications - irregular, wide and non-confluent calcium deposits which usually occur in large muscular and elastic arteries. These are components of the atherosclerotic disease $[15,16,20]$.

2. Media calcifications are smooth, circumferential granular deposits and involve small muscular arteries [20]. These usually involve the blood vessels in extremities, but can also involve the arteries of the breast, being identified easily by their typical appearance. These occur due to the ageing process $[15,16,20]$.

Mönckeberg medial calcific sclerosisis the calcium deposition in the media of the peripheral arterioles. It is also known as medial arterial calcification [21] and is an inci- dental finding in screening mammograms which are done for detection of early breast cancer in women above 40 years of age.

Typical appearance of BAC on mammography is described as linear, parallel lines [2], resembling a rail road appearance or as a ring-like appearance on cross section.

On ultrasound the atherosclerosis can be detected in the form of an increase in intima medial thickness (IMT). The IM thickening due to atherosclerosis in carotid vessels occurs due to involvement of the intima and media. However, the increase in IMT is not only related to the subclinical atherosclerosis but also can be the result of a non-atherosclerotic process such as hyperplasia of smooth muscle cells and fibrocellular hypertrophy. Therefore increased intima medial thickness is one of the important markers for atherosclerosis but it cannot be accepted as a risk factor for atherosclerosis and patients should not be subjected to treatment on its basis.

Although there are many studies emphasizing the correlation of BAC with cardiovascular diseases, studies showing an association between presence of BAC on 
mammography and carotid intima medial thickness have been limited. Therefore, this study was designed to test the hypothesis that BAC on mammography might be associated with C-IMT.

The C-IMT values of $\geq 0.85 \mathrm{~mm}$ and $<0.55 \mathrm{~mm}$ were reported as predictors of the presence and absence of CAD, respectively. Every $0.1 \mathrm{~mm}$ increase in baseline C-IMT was reported to increase future myocardial infarction and stroke risk by $10-15 \%$ and $13-18 \%$, respectively, whereas a $0.03 \mathrm{~mm} /$ year increase in C-IMT was reported to be related to a 2.2-fold increased risk of coronary events.

The difference between mean age in the two groups (BAC +ve and BAC -ve) was statistically significant in our study, which indicated that the prevalence of breast arterial calcification increases with age, which can be supported by the study done by Maas et al., who described aging as the major determinant of both types of vascular calcification (intima and medial type).
Yildiz et al. [17] found that age and postmenopausal duration showed a significant, independent relationship between the presence of BAC and C-IMT which is similar to our study.

Further, in our study we also correlated the grade of breast arterial calcification with the carotid intima medial thickness using Spearman's rho, which showed a non-significant positive correlation.

Erdogmus et al. [22] concluded that BAC positivity in patients with longer time after menopause could be attributed to the increase in age rather than the time after menopause; however, we found a positive correlation with the menopausal status.

\section{Conflict of interest}

The authors report no conflict of interest.

\section{References}

1. Markopoulos C, Mantas D, Revenas K, et al. Breast arterial calcifications as an indicator of systemic vascular disease. Acta Radiologica 2004; 45: 726-729.

2. Blackman G, Andreeva L, Coughlin B, et al. Breast arterial calcification on mammography: Incidence in various age groups. Radiology 2002.

3. Saxena A, Waddell I, Friesen R, Michalski R. Monckeberg medial calcific sclerosis mimicking malignant calcification pattern at mammography. J Clin Pathol 2005; 58: 447-448.

4. Rafieian-Kopaei M, Setorki M, Doudi M, et al. Atherosclerosis: process, indicators, risk factors and new hopes. Int J Prev Med 2014; 5: 927-946.

5. Gonzalez J, Wood JC, Dorey FJ, et al. Reproducibility of carotid intima-media thickness measurements in young adults. Radiology 2008; 247: 465-471.

6. Salonen JT, Salonen R. Ultrasonographically assessed carotid morphology and the risk of coronary heart disease. Arterioscler Thromb 1991; 11: 1245-1249.

7. Davis PH, Dawson JD, Mahoney LT, Lauer RM. Increased carotid intimal-medial thickness and coronary calcification are related in young and middle-aged adults: the Muscatine study. Circulation 1999; 100: 838-842.

8. Corrado E, Muratori I, Tantillo R, Contorno F. Relationship between endothelial dysfunction, intima media thickness and cardiovascular risk factors in asymptomatic subjects. Int Angiol 2005; 24: 52.

9. Salonen JT, Salonen R. Ultrasound B-mode imaging in observational studies of atherosclerotic progression. Circulation 1993; 87 (3 Suppl): II56-65.

10. Schnatz PF, Marakovits KA, O'Sullivan DM. The association of breast arterial calcification and coronary heart disease. Obstet Gynecol 2011; 117: 233-241

11. Cetin M, Cetin R, Tamer N. Prevalence of breast arterial calcification in hypertensive patients. Clin Radiol 2004; 59: 92-95.
12. Sickles E, Galvin H. Breast arterial calcification in association with diabetes mellitus: too weak a correlation to have clinical utility. Radiology 1985; 155: 577-579.

13. Yağtu M. Evaluating the association between breast arterial calcification and carotid plaque formation. J Breast Health 2015; 11: 180-185.

14. Yildiz S, Toprak H, Aydin S, et al. The association of breast arterial calcification and metabolic syndrome. Clinics 2014; 69: 841-846.

15. Duhn V, D’Orsi ET, Johnson S, et al. Breast arterial calcification: a marker of medial vascular calcification in chronic kidney disease. Clin J Am Soc Nephrol 2011; 6: 377-382.

16. Sedighi N, Radmard AR, Radmehr A, et al. Breast arterial calcification and risk of carotid atherosclerosis: focusing on the preferentially affected layer of the vessel wall. Eur J Radiol 2011; 79: 250-256.

17. Yildiz S, Yildiz A, Ertug N, et al. Association of breast arterial calcification and carotid intima-media thickness. Heart Vessels 2008; 23: $376-382$.

18. Maas AH, van der Schouw YT, Willem PTM, van der Graaf Y. Prevalence and determinants of breast arterial calcium in women at high risk of cardiovascular disease. Am J Cardiol 2004; 94: 655-659.

19. Tahmasebpour HR, Buckley AR, Cooperberg PL, Fix CH. Sonographic examination of the carotid arteries. Radiographics 2005; 25: 1561-1575.

20. Rotter MA, Schnatz PF, Currier Jr AA, O’Sullivan DM. Breast arterial calcifications (BACs) found on screening mammography and their association with cardiovascular disease. Menopause 2008; 15: 276-281.

21. Kataoka M, Warren R, Luben R, et al. How predictive is breast arterial calcification of cardiovascular disease and risk factors when found at screening mammography? Am J Roentgenol 2006; 187: 73-80.

22. Erdogmus B, Buyukkaya R, Buyukkaya A. Relationship between breast arterial calcifications detected on mammography and brachial artery intima-media thickness. Wien KlinWochenschr 2013; 125: $100-104$ 